Pacific Journal of Mathematics

ON A THEOREM DUE TO CASSEL S 


\title{
ON A THEOREM DUE TO CASSELS
}

\section{JosÉ M. Souto MENÉNDEZ}

\begin{abstract}
Using properties of one-dimensional formal groups, a proof is given of a theorem on the valuations of the torsion points of elliptic curves defined over $p$-adic fields.
\end{abstract}

1. Introduction. The aim of the present note is to give a proof of Theorem 5, due to Cassels, on the valuations of the torsion points of an elliptic curve defined over a local field $K$ of characteristic zero. Cassels's proof relies on the addition formulas for the Weierstrass $\wp$ and $\wp^{\prime}$ functions. The one given here follows from the properties of the torsion points of one-dimensional formal groups defined over the ring of integers of $K$.

The reader could also look at Oort [5] for another approach to Cassels' theorem.

2. Torsion points of formal groups. In the following we denote by $K$ a local field, finite extension of the field $Q_{p}$ of $p$-adic numbers, with ring of integers $A$; we assume that the normalized valuation $v$ of $K$ is extended to the algebraic closure $\bar{K}$ of $K$. We denote by $\mathfrak{p}_{K}$ (resp. $\mathfrak{p}_{\bar{K}}$ ) the maximal ideal of $A$ (resp. of the valuation ring of $\bar{K}$ ), and by $e=v(p)$ the ramification index of $K / Q_{P}$.

Let $F$ be a one-dimensional formal group of finite height $h \geq 1$, defined over $A$; as usual (see [3]), for each $a \in Z_{p}$ we denote by $[a](X) \in A[[X]]$ the unique endomorphism of $F$ such that $[a](X)=$ $a X+\cdots$. The group of points $F\left(\mathfrak{p}_{\bar{K}}\right)$ of $F$ with values in $\bar{K}$ has a structure of a module over $Z_{p}$, by means of the operation $a \cdot x=[a](x)$, $a \in Z_{p}, x \in F\left(\mathfrak{p}_{\bar{K}}\right) ; F\left(\mathfrak{p}_{K}\right)$ is a sub- $Z_{p}$-module of $F\left(\mathfrak{p}_{\bar{K}}\right)$.

Let $[p](X)=\sum_{i=1}^{\infty} a_{i} X^{i}\left(a_{1}=p\right)$ be the "multiplication by $p$ " in the formal group $F$; setting $q=p^{h}$, one has $a_{i} \in \mathfrak{p}_{K}$ if $i=1, \ldots, q-$ 1 and $v\left(a_{q}\right)=0$. We shall be interested in the valuations of the torsion points $x \in F\left(p_{\bar{K}}\right)$. The most convenient thing is to consider the Newton polygon of the series $[p](X)$, that is the lower convex envelope of the points $\left(i, v\left(a_{i}\right)\right) \in R^{2}(i \geq 1)$.

If $P_{0}=(1, e), P_{1}=\left(q_{1}, e_{1}\right), \ldots, P_{m}=(q, 0)$ are the vertices of such a polygon (where $e_{i}=v\left(a_{q_{1}}\right)$ ), the slopes are the negative of the numbers 
$\alpha_{1}=\left(e-e_{1}\right) /\left(q_{1}-1\right), \ldots, \alpha_{m}=e_{m-1} /\left(q_{m}-q_{m-1}\right)\left(\alpha_{1}>\alpha_{2}>\cdots>\right.$ $\alpha_{m}$ ). If $q_{i} \leq r \leq q_{i+1}$ (for $i=0, \ldots, m-1$ ), for any $x \in \mathfrak{p}_{\bar{K}}$ one has $v\left(a_{r} x^{r}\right) \geq \inf \left(v\left(a_{q_{i}} x^{q_{l}}\right), v\left(a_{q_{t+1}} x^{q_{t+1}}\right)\right)$; moreover, if $r>q_{m}=q$, for $x \in \mathfrak{p}_{\bar{K}}, v\left(a_{r} x^{r}\right)>v\left(a_{q} x^{q}\right)$. Therefore, for any $x \in \mathfrak{p}_{\bar{K}}$ with $[p](x)=0$, there exists $i=0, \ldots, m-1$ such that $v\left(a_{q_{l}} x^{q_{i}}\right)=v\left(a_{q_{t+1}} x^{q_{t+1}}\right)$, so that $v(x)=\alpha_{i+1}$. Moreover (see Koblitz [4]) the number of roots $x \in \mathfrak{p}_{\bar{K}}$ of the series $[p](X)$, of valuation $\alpha_{i+1}$, is $q_{i+1}-q_{i}$.

LEMMA 1. With the above notations, the $q_{i}$ are powers of $p$.

Proof. For each $i=1, \ldots, m$, the set

$$
\left\{x \in F\left(\mathfrak{p}_{\bar{K}}\right) \mid[p](x)=0, v(x) \geq \alpha_{i}\right\}
$$

is an elementary abelian $p$-group (with the operation given by the formal group law $F)$; as its order is $\left(q_{i}-q_{i-1}\right)+\cdots+\left(q_{1}-1\right)+1$, the lemma is obvious.

Proposition 2. For any $x \in \mathfrak{p}_{\bar{K}}$, one has

-if $v(x)<\alpha_{m}$, then $v([p](x))=q v(x)$,

-if $\alpha_{i+1}<v(x)<\alpha_{i}$, then $v([p](x))=e_{i}+q_{i} v(x)$,

-if $\alpha_{1}<v(x)$, then $v([p](x))=e+v(x)$.

Proof. For $x \in F\left(\mathfrak{p}_{\bar{K}}\right)$ such that $v(x)<\alpha_{m}$, then for any $r \neq q_{m}=q$, $v\left(a_{q} x^{q}\right)<v\left(a_{r} x^{r}\right)$. In fact, when $r>q$ such a relation is obvious (since $v\left(a_{q}\right)=0$ ); when $r<q$, one may write

$$
\begin{aligned}
v(x)<\alpha_{m} & =\left(v\left(a_{q_{m-1}}\right)-v\left(a_{q}\right)\right) /\left(q-q_{m-1}\right) \\
& <\left(v\left(a_{r}\right)-v\left(a_{q}\right)\right) /(q-r),
\end{aligned}
$$

hence $v\left(a_{q} x^{q}\right)<v\left(a_{r} x^{r}\right)$.

If $\alpha_{i+1}<v(x)<\alpha_{i}$ (with $i=1, \ldots, m-1$ ), then for any $r \neq q_{i}$, one has $v\left(a_{q_{i}} x^{q_{t}}\right)<v\left(a_{r} x^{r}\right)$. In fact, for $r>q_{i}$ this relation comes from

$$
v(x)>\left(v\left(a_{q_{1}}\right)-v\left(a_{q_{1+1}}\right)\right) /\left(q_{i+1}-q_{i}\right) \geq\left(v\left(a_{q_{1}}\right)-v\left(a_{r}\right)\right) /\left(r-q_{i}\right) ;
$$

for $r<q_{i}$, it comes from

$$
v(x)<\left(v\left(a_{q_{i-1}}\right)-v\left(a_{q_{1}}\right)\right) /\left(q_{i}-q_{i-1}\right) \leq\left(v\left(a_{r}\right)-v\left(a_{q_{1}}\right)\right) /\left(q_{i}-r\right) .
$$

The case $v(x)>\alpha_{1}$ is discussed similarly.

REMARKS. (1) If $v(x)=\alpha_{i},[p](x) \neq 0$ (for $i=1, \ldots, m$ ), arguing as above, one gets $v([p](x)) \geq e_{i}+q_{i} \alpha_{i}$.

(2) For $i>\alpha_{1}, x \rightarrow[p](x)$ induces an isomorphism $F\left(\mathfrak{p}_{K}^{i}\right) \rightarrow$ $F\left(\mathfrak{p}_{K}^{i+e}\right)$ (of course, we denote by $F\left(\mathfrak{p}_{K}^{r}\right)$ the set $\mathfrak{p}_{K}^{r}$ with the group 
structure given by the group law $F$ ). The injectivity comes from the fact that in $\mathfrak{p}_{\bar{K}}$ the zeros of $[p](X)$ have valuation $\leq \alpha_{1}$. To show the surjectivity, let $\Pi$ be a uniformizing parameter of $K$; we have to see that if $y \in \mathfrak{p}_{K}$ is such that $v(y)=i+e>\alpha_{1}+e$, there is $x=\Pi^{\alpha_{1}} t(t \in A)$ such that $[p]\left(\Pi^{\alpha_{1}} t\right)=y$; now, the series

$$
\frac{1}{\Pi^{\alpha_{1}+e}}\left(-y+p \Pi^{\alpha_{1}} T+a_{2} \Pi^{2 \alpha_{1}} T^{2}+\cdots\right)
$$

has coefficients in $A$ and Weierstrass degree one, so the result follows from the Preparation Theorem for power series.

(3) If $F$ is the multiplicative group, the Newton polygon of $[p](X)$ only has one slope. Proposition 2 gives then the well-known effect of "raising to the $p$ th power" in the group of principal units of the local field $K$ (or of any of its finite extensions).

Proposition 3. $F\left(\mathfrak{p}_{K}\right)$ is a $Z_{p}$-module of finite type, whose rank modulo torsion is $\left[K: Q_{p}\right]$. The torsion subgroup is a finite p-group.

Proof. For each $i \geq 1$, let us denote, as above, by $F\left(\mathfrak{p}_{K}^{i}\right)$ the abelian group on the set $\mathfrak{p}_{K}^{i}$ with the operation given by $(x, y) \rightarrow F(x, y)$; of course, $F\left(\mathfrak{p}_{K}^{i}\right)$ is a $Z_{p}$-submodule of $F\left(\mathfrak{p}_{K}\right)$. It is trivial that $\mathfrak{p}_{K}^{i} / \mathfrak{p}_{K}^{i+1} \simeq$ $F\left(\mathfrak{p}_{K}^{i}\right) / F\left(\mathfrak{p}_{K}^{i+1}\right)$.

The filtration $F\left(\mathfrak{p}_{K}\right) \supset F\left(\mathfrak{p}_{K}^{2}\right) \supset \cdots$ is separated and produces in $F\left(\mathfrak{p}_{K}\right)$ the $p$-adic topology (if $i$ is large enough, one of the remarks shows that $\left.p F\left(\mathfrak{p}_{K}^{i}\right)=F\left(\mathfrak{p}_{K}^{i+e}\right)\right)$. According to a well-known lemma in commutative algebra, the finiteness of $F\left(\mathfrak{p}_{K}\right)$ as a module over $Z_{p}$, follows from the finiteness of $F\left(\mathfrak{p}_{K}\right) / p F\left(\mathfrak{p}_{K}\right)$, a quotient of $F\left(\mathfrak{p}_{K}\right) / F\left(\mathfrak{p}_{K}^{i+e}\right)=F\left(\mathfrak{p}_{K}\right) / p F\left(\mathfrak{p}_{K}^{i}\right)$ for $i$ large enough.

Taking again $i$ large enough so that $F\left(\mathfrak{p}_{K}^{i}\right)$ is torsion free, hence free, its rank is the same as $\operatorname{dim}_{F_{p}}\left(F\left(\mathfrak{p}_{K}^{i}\right) / p F\left(\mathfrak{p}_{K}^{i}\right)\right)=\operatorname{dim}_{F_{p}}\left(F\left(\mathfrak{p}_{K}^{i}\right) / F\left(\mathfrak{p}_{K}^{i+e}\right)\right)$; since $\left(F\left(\mathfrak{p}_{K}^{i}\right): F\left(\mathfrak{p}_{K}^{i+e}\right)\right)=p^{\left[K: Q_{p}\right]}$, the proposition is clear.

Proposition 4. Let $x \in F\left(p_{\bar{K}}\right)$ be a torsion point of order $p^{r}$. Then $v(x) \leq e / \varphi\left(p^{r}\right)=e / p^{r-1}(p-1)$.

Proof. From Proposition 2, it is obvious that for any $x \in F\left(\mathfrak{p}_{\bar{K}}\right)$, $v([p](x)) \geq v(x)$; therefore, if $v(x)>\alpha_{1}, x$ is not a torsion point.

One proves the proposition by induction on $r$. If $x$ is of order $p$, $v(x) \leq \alpha_{1}=\left(e-e_{1}\right) /\left(q_{1}-1\right) \leq e /(p-1)$ (by Lemma 1). If $x$ is of order $p^{r}(r>1)$, then $v(x) \leq \alpha_{1}$ and $v([p](x)) \leq e / p^{r-2}(p-1)$, by 
the induction hypothesis; again by Proposition 2,

$$
v(x) \leq \alpha_{1} \Rightarrow v([p](x)) \geq p v(x),
$$

so $v(x) \leq v([p](x)) / p \leq e / p^{r-1}(p-1)$.

Remark. Sometimes, one can be more precise about $v(x)$. If the height of $F$ is $h=1$, the Newton polygon has only one slope and all the points of order $p$ have valuation $e /(p-1)$; in this case, if $x \in F\left(p_{\bar{K}}\right)$ is of order $p^{r}(r \geq 1), v(x)=e / p^{r-1}(p-1)$.

If the height of $F$ is $h=2$, there are two possibilities for the Newton polygon. If there is only one slope, the points $x \in F\left(\mathfrak{p}_{\bar{K}}\right)$ of order $p^{r}$ have exact valuation $v(x)=e / p^{2(r-1)}\left(p^{2}-1\right)$. If there are two slopes, one cannot say more than in Proposition 4.

3. Cassels's theorem. In the following theorem, $E$ denotes an elliptic curve defined over the local field $K$, given by a minimal Weierstrass equation

$$
y^{2}+a_{1} x y+a_{3} y=x^{3}+a_{2} x^{2}+a_{4} x+a_{6}
$$

$\left(a_{i} \in A\right)$. We write $E(K)$ for the group of points of $E$ with values in $K$; $E(K)$ is an abelian group in the usual way, taking the point at infinity $(0,1,0)$ of $E$ as zero element. Notations are the same as in Tate [6].

TheOREM 5. Let $(x, y) \in E(K)$ a torsion point of $E$. If the order of $(x, y)$ is not a power of $p$, then $x, y \in A$. If the order of $(x, y)$ is $p^{r}(r \geq 1)$, then

$$
v(x) \geq-2 e / p^{r-1}(p-1), \quad v(y) \geq-3 e / p^{r-1}(p-1) .
$$

Proof. By reducing the equation (X) of $E$ modulo the maximal ideal of $A$, we get the equation of a cubic $\tilde{E}$ defined over the residue field $k$ of $K$. The set $\tilde{E}_{n s}(k)$ of nonsingular points of $\tilde{E}$ with values in $k$ is a group, and one has the exact sequence

$$
0 \rightarrow E_{1}(K) \rightarrow E_{0}(K) \rightarrow \tilde{E}_{n s}(k) \rightarrow 0 ;
$$

here $E_{0}(K)$ denotes the subgroup of the elements of $E(K)$ that reduce to the nonsingular points of $E$, and

$$
E_{1}(K)=0 \cup\{(x, y) \in E(K) \mid v(x) \leq-2, v(y) \leq-3\}
$$

is the kernel of the reduction map. 
One knows that there is a formal group law $F$ defined over $A$, and an isomorphism

$$
\begin{aligned}
E_{1}(K) & \stackrel{\sim}{\rightarrow} F\left(\mathfrak{p}_{K}\right), \\
(x, y) & \rightarrow-x / y .
\end{aligned}
$$

Such a formal group $F$ is isomorphic to the additive one if $E$ has bad reduction and the singularity of $\tilde{E}$ is a cusp, and of height one or two in the other cases; in the first case, $F\left(\mathfrak{p}_{K}\right)$ is of course torsion free, and in the other ones, the only possible torsion is $p$-torsion. In these cases, if $z \in F\left(\mathfrak{p}_{K}\right)$ has order $p^{r}, v(z) \leq e / p^{r-1}(p-1)$ (Proposition $4)$; since we have for the corresponding point $(x, y) \in E_{1}(K)$

$$
\begin{aligned}
& x=z^{-2}-a_{1} z^{-1}-a_{2}-\cdots, \\
& y=-z^{-1} x,
\end{aligned}
$$

we get $v(x)=-2 v(z) \geq-2 e / p^{r-1}(p-1), v(y) \geq-3 e / p^{r-1}(p-1)$.

The theorem is proved taking account of the fact that

$$
E(K)-E_{1}(K)=\{(x, y) \in E(K) \mid x, y \in A\}
$$

COROLlARY 6 (Nagell-Lutz). Let $E$ be an elliptic curve defined over $Q$, given by a minimal global Weierstrass equation of the form $(\mathrm{X})$ with the $a_{i}$ rational integers. Then the torsion points of $E(Q)$ have integer coordinates, with one possible exception: there could be a unique point of order two of the form $(a / 4, b / 8)$, with $a, b, \in Z$.

Proof. For each prime number $p$, we denote by $v_{p}$ the $p$-adic valuation of $Q$ (extended to $Q_{p}$ ). Since we have $E(Q) \subset E\left(Q_{p}\right)$, we can apply the last theorem.

If $(x, y) \in E(Q)$ is a torsion point whose order is not a power of any prime number $p$, then $x, y \in Z_{p}$ for each $p$, so $x, y \in Z$.

If the order of $(x, y) \in E(Q)$ is $p^{r}$ ( $p$ prime, $r \geq 1$ ), then for each prime $l \neq p, x, y \in Z_{l}$; moreover

$$
v_{p}(x) \geq-2 / p^{r-1}(p-1), \quad v_{p}(y) \geq-3 / p^{r-1}(p-1),
$$

so $x, y \in Z_{p}$ unless, perhaps, $p^{r}=2,3$, 4 . If $p^{r}=3$ or 4 , again $x, y \in Z_{p}$, since $x, y \notin Z_{p}$ implies $v_{p}(x) \leq-2, v_{p}(y) \leq-3$.

So we are only left with the possibility of points of order $p^{r}=2$; if $(x, y) \in E(Q)$ is one of those points, $x, y \in Z_{l}$ for each $l \neq 2$ and $\nu_{2}(x)=-2, v_{2}(y)=-3$; then $(x, y)$ should belong to the kernel $E_{l}\left(Q_{2}\right)$ of the reduction of $E$ modulo 2. Looking at the power series [2] $(X)=2 X-a_{1} X^{2}-2 a_{2} X^{3}+\cdots$, we find that, in fact, if the formal 
group $F$ associated to the model (X) of the curve $E$ is of height one in $Z_{2}\left(\Longleftrightarrow a_{1} \notin 2 Z\right)$, there exists in $E\left(Q_{2}\right)$ a unique point of order two whose coordinates are $(a / 4, b / 8), a, b \in Z_{2}$; such a point may or may not be in $E(Q)$.

REMARK. As shown in the proof, one has to study the possibility of a torsion point of order two in $E(Q)$ only when $E$ has ordinary good reduction or split multiplicative reduction at 2 .

4. Appendix. If $P=(x(P), y(P)) \in E(Q)$ is a torsion point of order different from two of the curve $E$ given by the equation $(\mathrm{X})-$ where the $a_{i} \in Z$-we know that $x(P), y(P) \in Z$, and so $P$ verifies the hypothesis of the following proposition.

Proposition 7. Let $\Delta$ be the discriminant of the curve $E$. If $P=(x(P), y(P)) \in E(Q)$ is a point with integer coordinates such that $2 P=(x(2 P), y(2 P))$ has also integer coordinates, then $\left(2 y(P)+a_{1} x(P)+a_{3}\right)^{2} \mid \Delta$.

Proof. We only sketch it. We write, as in [6],

$$
\begin{aligned}
b_{2} & \doteq a_{1}^{2}+4 a_{2}, \quad b_{4}=a_{1} a_{3}+2 a_{4}, \quad b_{6}=a_{3}^{2}+4 a_{6}, \\
b_{8} & =a_{1}^{2} a_{6}-a_{1} a_{3} a_{4}+4 a_{2} a_{6}+a_{2} a_{3}^{2}-a_{4}^{2}, \\
\Delta & =-b_{2}^{2} b_{8}-8 b_{4}^{3}-27 b_{6}^{2}+9 b_{2} b_{4} b_{6} .
\end{aligned}
$$

Multiplication by two, $E \stackrel{2}{\rightarrow} E$, is given by a formula

$$
2(x, y)=\left(x_{2}, y_{2}\right)
$$

where $x_{2}=u(x) / f(x)$, with $u(T)=T^{4}-b_{4} T^{2}-2 b_{6} T-b_{8}$ and $f(T)=4 T^{3}+b_{2} T^{2}+2 b_{4} T+b_{6}$; one has $\operatorname{disc}_{3}(f(T))=16 \Delta$, and the relation

$$
16 u(T)-f^{\prime}(T)^{2}+4\left(8 T+b_{2}\right) f(T)=0 .
$$

One verifies, with the notations of Bourbaki [1] (Ch. IV, §6),

$$
\begin{aligned}
\operatorname{Res}_{4,3}(16 u(T), f(T)) & =\operatorname{Res}_{4,3}\left(f^{\prime}(T)^{2}-4\left(8 T+b_{2}\right) f(T), f(T)\right) \\
& =\operatorname{Res}_{4,3}\left(f^{\prime}(T)^{2}, f(T)\right)=\left[\operatorname{Res}_{2,3}\left(f^{\prime}(T), f(T)\right)\right]^{2} \\
& =16\left(\operatorname{disc}_{3} f(T)\right)^{2}=2^{12} \Delta^{2}
\end{aligned}
$$

therefore, $\operatorname{Res}_{4,3}(u(T), f(T))=\Delta^{2}$. 
On the other hand

$\operatorname{Res}_{4,3}(u(T), f(T))$

$=\left|\begin{array}{ccccccc}1 & 0 & 0 & 4 & 0 & 0 & 0 \\ 0 & 1 & 0 & b_{2} & 4 & 0 & 0 \\ -b_{4} & 0 & 1 & 2 b_{4} & b_{2} & 4 & 0 \\ -2 b_{6} & -b_{4} & 0 & b_{6} & 2 b_{4} & b_{2} & 4 \\ -b_{8} & -2 b_{6} & -b_{4} & 0 & b_{6} & 2 b_{4} & b_{2} \\ 0 & -b_{8} & -2 b_{6} & 0 & 0 & b_{6} & 2 b_{4} \\ 0 & 0 & -b_{8} & 0 & 0 & 0 & b_{6}\end{array}\right|$

$\begin{aligned} & \left|\begin{array}{ccccccc}1 & 0 & 0 & 4 & 0 & 0 & 0 \\ 0 & 1 & 0 & b_{2} & 4 & 0 & 0 \\ -b_{4} & 0 & 1 & 2 b_{4} & b_{2} & 4 & 0 \\ -2 b_{6} & -b_{4} & 0 & b_{6} & 2 b_{4} & b_{2} & 4 \\ -b_{8} & -2 b_{6} & -b_{4} & 0 & b_{6} & 2 b_{4} & b_{2} \\ 0 & -b_{8} & -2 b_{6} & 0 & 0 & b_{6} & 2 b_{4} \\ T^{2} u(T) & T u(T) & u(T) & T^{3} f(T) & T^{2} f(T) & T f(T) & f(T)\end{array}\right| \\ = & -48 \Delta T^{2} u(T)-8 b_{2} \Delta T u(T)+\left(b_{2}^{2}-32 b_{4}\right) \Delta u(T)+12 \Delta T^{3} f(T)\end{aligned}$

here we have developed the last determinant by the last row, and made systematic use of the relation $4 b_{8}=b_{2} b_{6}-b_{4}^{2}$.

Therefore,

$$
\begin{aligned}
\Delta= & \left(-48 T^{2}-8 b_{2} T+\left(b_{2}^{2}-32 b_{4}\right)\right) u(T) \\
& +\left(12 T^{3}-b_{2} T^{2}-10 b_{4} T+\left(b_{2} b_{4}-27 b_{6}\right)\right) f(T) .
\end{aligned}
$$

Now, if $P=(x(P), y(P)) \in E(Q)$ and $2 P=(x(2 P), y(2 P))$ have integer coordinates, as $u(x(P))=x(2 P) f(x(P))$, we get

$$
f(x(P)) \mid \Delta \text {. }
$$

Since $y^{2}+a_{1} x y+a_{3} y=x^{3}+a_{2} x^{2}+a_{4} x+a_{6}$ implies

$$
\left(2 y+a_{1} x+a_{3}\right)^{2}=4 x^{3}+b_{2} x^{2}+2 b_{4} x+b_{6}=f(x),
$$

the proposition is proved. 


\section{REFERENCES}

[1] N. Bourbaki, Algèbre, Chapitre 4 á 7. Ed. Masson, 1981.

[2] J. W. S. Cassels, Diophantine equations with special reference to elliptic curves, J. London Math. Soc., 41 (1966), 193-291.

[3] A. Frohlich, Formal Groups, Lecture Notes in Math. 74, Springer 1968.

[4] N. Koblitz, p-adic Numbers, p-adic Analysis and Zeta Functions, Graduate Texts in Mathematics 58, Springer 1977.

[5] F. Oort, Elliptic curves: Diophantine torsion solutions and singular $j$-invariants, Math. Ann., 207, 139-162.

[6] J. Tate, The arithmetic of elliptic curves, Inv. Math., 23 (1974), 179-206.

Received May 16, 1985.

Perlora

Carreño

Asturias, SpaIN 


\section{PACIFIC JOURNAL OF MATHEMATICS}

\section{EDITORS}

\author{
V. S. VARADARAJAN \\ (Managing Editor) \\ University of California \\ Los Angeles, CA 90024 \\ HeRBERT ClEMENS \\ University of Utah \\ Salt Lake City, UT 84112 \\ R. FINN \\ Stanford University \\ Stanford, CA 94305
}

\section{HERMANN FLASCHKA \\ University of Arizona \\ Tucson, AZ 85721}

VAUghan F. R. Jones

University of California

Berkeley, CA 94720

ROBION KIRBY

University of California

Berkeley, CA 94720

\author{
C. C. MOORE \\ University of California \\ Berkeley, CA 94720
}

HAROLD STARK

University of California, San Diego

La Jolla, CA 92093

\section{ASSOCIATE EDITORS}
R. ARENS
E. F. BECKENBACH
B. H. NEUMANN
F. WOLF
K. YOSHIDA
(1906-1982)

\section{SUPPORTING INSTITUTIONS}

\begin{abstract}
UNIVERSITY OF ARIZONA
UNIVERSITY OF BRITISH COLUMBIA

UNIVERSITY OF CALIFORNIA

MONTANA STATE UNIVERSITY

UNIVERSITY OF NEVADA, RENO

NEW MEXICO STATE UNIVERSITY

OREGON STATE UNIVERSITY
\end{abstract}
CALIFORNIA INSTITUTE OF TECHNOLOGY

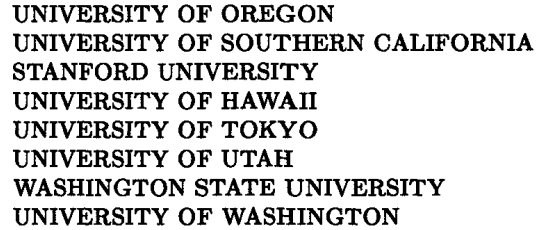

The Supporting Institutions listed above contribute to the cost of publication of this Journal, but they are not owners or publishers and have no responsibility for its content or policies.

Mathematical papers intended for publication in the Pacific Journal of Mathematics should be in typed form or offset-reproduced (not dittoed), double spaced with large margins. Please do not use built up fractions in the text of the manuscript. However, you may use them in the displayed equations. Underline Greek letters in red, German in green, and script in blue. The first paragraph must be capable of being used separately as a synopsis of the entire paper. In particular it should contain no bibliographic references. Please propose a heading for the odd numbered pages of less than 35 characters. Manuscripts, in triplicate, may be sent to any one of the editors. Please classify according to the scheme of Math. Reviews, Index to Vol. 39. Supply name and address of author to whom proofs should be sent. All other communications should be addressed to the managing editor, or Elaine Barth, University of California, Los Angeles, California 90024.

There are page-charges associated with articles appearing in the Pacific Journal of Mathematics. These charges are expected to be paid by the author's University, Government Agency or Company. If the author or authors do not have access to such Institutional support these charges are waived. Single authors will receive $\mathbf{5 0}$ free reprints; joint authors will receive a total of 100 free reprints. Additional copies may be obtained at cost in multiples of 50 .

The Pacific Journal of Mathematics is issued monthly as of January 1966. Regular subscription rate: $\$ 190.00$ a year (5 Vols., 10 issues). Special rate: $\$ 95.00$ a year to individual members of supporting institutions.

Subscriptions, orders for numbers issued in the last three calendar years, and changes of address should be sent to Pacific Journal of Mathematics, P.O. Box 969, Carmel Valley, CA 93924, U.S.A. Old back numbers obtainable from Kraus Periodicals Co., Route 100, Millwood, NY 10546.

The Pacific Journal of Mathematics at P.O. Box 969, Carmel Valley, CA 93924 (ISSN 0030-8730) publishes 5 volumes per year. Application to mail at Second-class postage rates is pending at Carmel Valley, California, and additional mailing offices. Postmaster: send address changes to Pacific Journal of Mathematics, P.O. Box 969, Carmel Valley, CA 93924.

PUBLISHED BY PACIFIC JOURNAL OF MATHEMATICS, A NON-PROFIT CORPORATION Copyright (C) 1988 by Pacific Journal of Mathematics 


\section{Pacific Journal of Mathematics}

Vol. 135, No. $1 \quad$ September, 1988

Margaret M. Bayer, Barycentric subdivisions $\ldots \ldots \ldots \ldots \ldots \ldots \ldots \ldots \ldots$

Eung Chun Cho, $s$-Smith equivalent representations of dihedral groups . . . . 17

Avner Friedman and Jindrich Necas, Systems of nonlinear wave equations

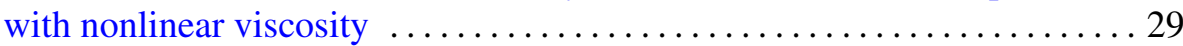

Kyong Taik Hahn, Nontangential limit theorems for normal mappings . . . . 557

Eloise A. Hamann, Evan Green Houston, Jr. and Jon Lee Johnson,

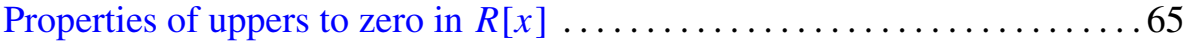

José M. Souto Menéndez, On a theorem due to Cassels ............. 81

Courtney Hughes Moen, Irreducibility of unitary principal series for

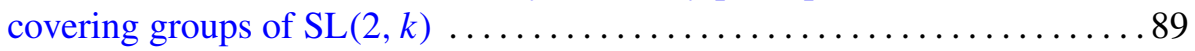

Frank M. Neubrander, Integrated semigroups and their applications to the

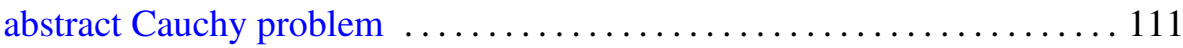

C. K. Qu and Roderick Sue-Chuen Wong, Szegő's conjecture on Lebesgue

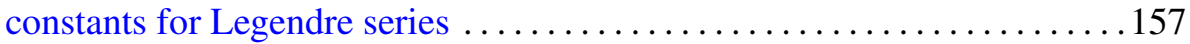

John Brendan Sullivan, The Euler character and cancellation theorems for

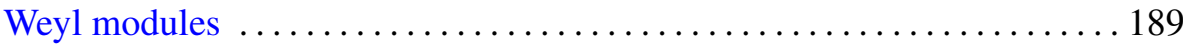

